\title{
Die Problematik in den schweizerischen Uhrenregionen oder Forderung nach einer innovationsorientierten Regionalpolitik
}

\section{NEUARTIGER REGIONALPOLITISCHER PROBLEMDRUCK: DAS BEISPIEL DER UHRENREGIONEN}

\section{Deutliche Alarmzeichen}

Ein wirtschaftlich ausserordentlich dynamischer Wirtschaftsraum ( $v g l$. Abb.) mit einer überdurchschnittlichen Erwerbsquote von $49,7 \%$ im Jahre 1970 (Schweiz: 47,9\%), mit einem dominierenden industriell-gewerblichen Sektor, der 1970 63\% der Arbeitnehmer heschäftigte (Schweiz: $49 \%$ ), mit einer vorherrschenden Branche (Uhrenindustrie), die jahrzehntelang eine monopolähnliche Weltmarktstellung besass, kurz: eine im Rahmen der schweizerischen Volkswirtschaft überdurchschnittlich kräftige Region kcmmt plötzlich, innert wenigen Jahren in grosse tiefgreifende Schwierigkeiten:

- Sie verliert in der Zeit von 1970 - 1976 $44 ' 488$ industriell-gewerbliche oder $28,4 \%$ ihrer Arbeitsplätze, knapp 3/4 davon erst nach 1974;

- der Anteil der Uhrenindustrie an den regionalen Arbeitsplätzen sinkt gesamthaft von $38,7 \%(1970)$ auf $34,9 \%(1976)$;

- der Anteil an ausländischen Arbeitskräften steigt in der gleichen Phase vcrerst von $26 \%$ (1970) auf 31\% (1974), um dann schlagartig auf $23,5 \%$ (1976) zurückzusinken;

- die Bevölkerung nimmt in der Zeit von 1970 1976 erheblich ab, Schätzungen lauten auf rund $-15 \%$.

Solchen und weiteren Alarmzeichen müssen kräftig wirksame Mechanismen zugrundeliegen, die einen tiefgreifenden wirtschaftlichen Strukturwandel in diesen Regionen induziert haben.

Eine Hauptursache: die Uhrenindustrie in Bedrängnis

Rund die Hälfte (48\%) des beschriebenen Verlustes an industriell-gewerblichen Arbeitsplätzen geht auf das Konto der Uhrenindustrie, die in einigen Regionen - beispielsweise in La Vallée, Solothurn III, Centre-Jura, Jura und Biel (vgl. Abb.) - noch 1976 die absolut dominierende Branche mit einem Anteil von nahezu oder über $50 \%$ an allen industriell-gewerblichen Arbeitsplätzen ist (1).

Ein weiteres Drittel (35\%) des gesamten Verlustes geht auf das Konto der Maschinen-, Apparate- und Metallindustrie und damit der Uhrendindustrie in diesen Regionen nah verwandte Branchen, die in einem dichten Netz von $\mathrm{Zu}$ - und Ablieferungsbeziehungen mit ihr verbunden sind (2).

Die Probleme der Uhrenregionen können somit in erster Linie auf die Probleme der Uhrenindustrie zurückgeführt werden.

Diese Branche besitzt auch gegenwärtig noch einen erheblichen Stellenwert in der schweizerischen Volkswirtschaft:

sie beschäftigt 1975 rund $5 \%$ aller industriellen Arbeitskräfte und führt Produkte im Umfang von rund $6 \%$ der gesamten wertmässigen Exporte der Schweiz aus.

Für die Uhrenregionen von ausschlaggebender Bedeutung ist allerdings die für sie nachteilige Entwick?ung dieses Stellenwertes:

- Die Zahl der Beschäftigten in der Uhrenindustrie ist von $89^{\prime} 448$ im Jahre 1970 auf $55^{\prime} 18$ ? (1976) und schliesslich auf rund 45'000 im März 1978 (Schätzung) gesunken. Innert 7 Jahren hat diese Branche demzufolge jeden zweiten Arbeitsplatz aufgegeben, den Grossteil davon nach 1974.

- Die Entwicklung der internationalen Wettbewerbsstellung der Branche lässt sich am Ant€il an der mengenmässigen Weltproduktion ablesen. Dieser Anteil sank von $42 \%$ im Jahre 1970 auf 38\% 1974 und schliesslich auf 28\% Ende 1976. Noch 1950 betrug er knapp über $50 \%$. Auch in Zeiten absolut ansteigender Exporte (bis 1974) sind somit markante Marktverluste eingetreten.

Den Gründen für diese Verluste an Weltmarktanteilen kann in diesem Artikel nur stichwortartig nachgegangen werden (3); sie können in der Veränderung äusserer Rahmenbedingungen oder in inneren, vorwiegend branchenstrukturellen Ursachen verankert sein.

Als externe Ursachen lassen sich schlagwortartig aufführen:

- Importbeschränkungen zahlreicher Konkurrenzländer und damit eine markante Verzerrung des internationalen Wettbewerbes;

- relativ hohes schweizerisches Lohnkostenniveau, welches sich direkt in den Produktionskosten niederschlägt (die Lohnkosten in gewissen Ländern Südostasiens betragen nur $10-20 \%$ der schweizerischen;

- die Aufwertung des Schweizer Frankens, welche zwischen 1972 und 1976 eine paritätsbedingte Preissteigerung für Schweizer Uhren von 20 -

Dr. Ernst A. Brugger, Zihlgasse, 3204 Rosshäusern 


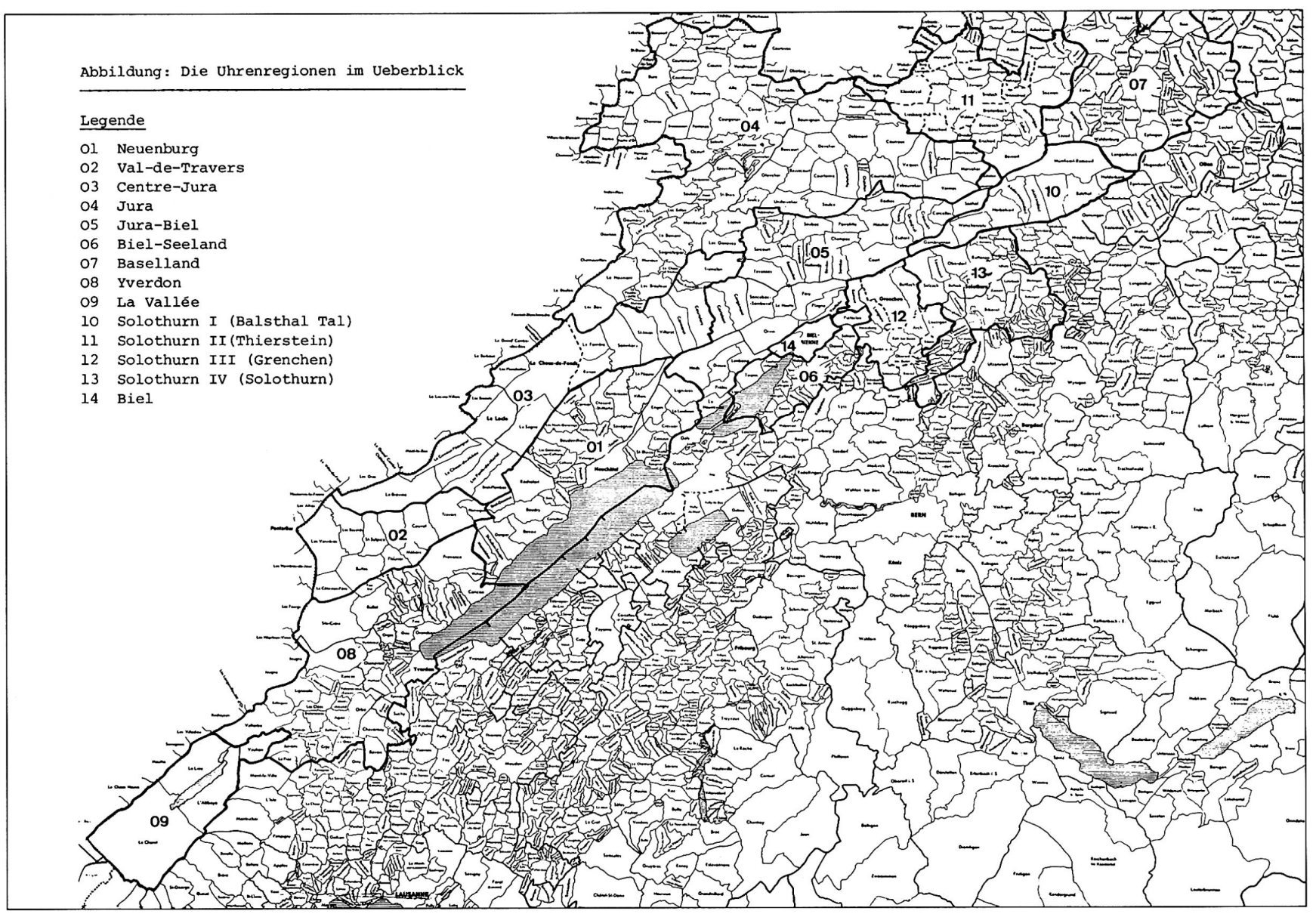


$25 \%$, seither wahrscheinlich nochmals eine um rund $20 \%$ verursachte;

- das Aufkommen der Elektronik auch für die Uhrenproduktion und damit eine Verunsicherung im technischen Bereich und im gesamten Marketing.

Gesamthaft erklären diese externen Faktoren einen Teil des Rückschlages, nicht jedoch seine Langfristigkeit und seine Tiefe.

Hiefür ist vielmehr ein internes Ursachenbündel verantwortlich, das mit dem Stichwort "Anpassungsschwierigkeiten" vorläufig abstrakt umschrieben sei. Wir kommen darauf zurück.

Notwendigkeit einer differenzierten Betrachtungsweise

Von entscheidender Bedeutung für die Regionen der Jurakette ist jedoch die Tatsache, dass die geschilderte Problematik nicht für alle Produktionslinien der Branche in gleichem Ausmass zutrifft (4) und dass diese Produktionslinien mit ihrem spezifischen Problemgehalt zudem regional unterschiedlich konzentriert auftreten (5). Hinzu kommt die von Betrieb zu Betrieb unterschiedliche Fähigkeit zur Anpassung an veränderte Marktdaten und zur Realisierung strukturfördender einzelbetrieblicher Massnahmen (6).

Tab. 1: Klassierung der Regionen nach Kriterien inbezug auf die Uhrenbranche

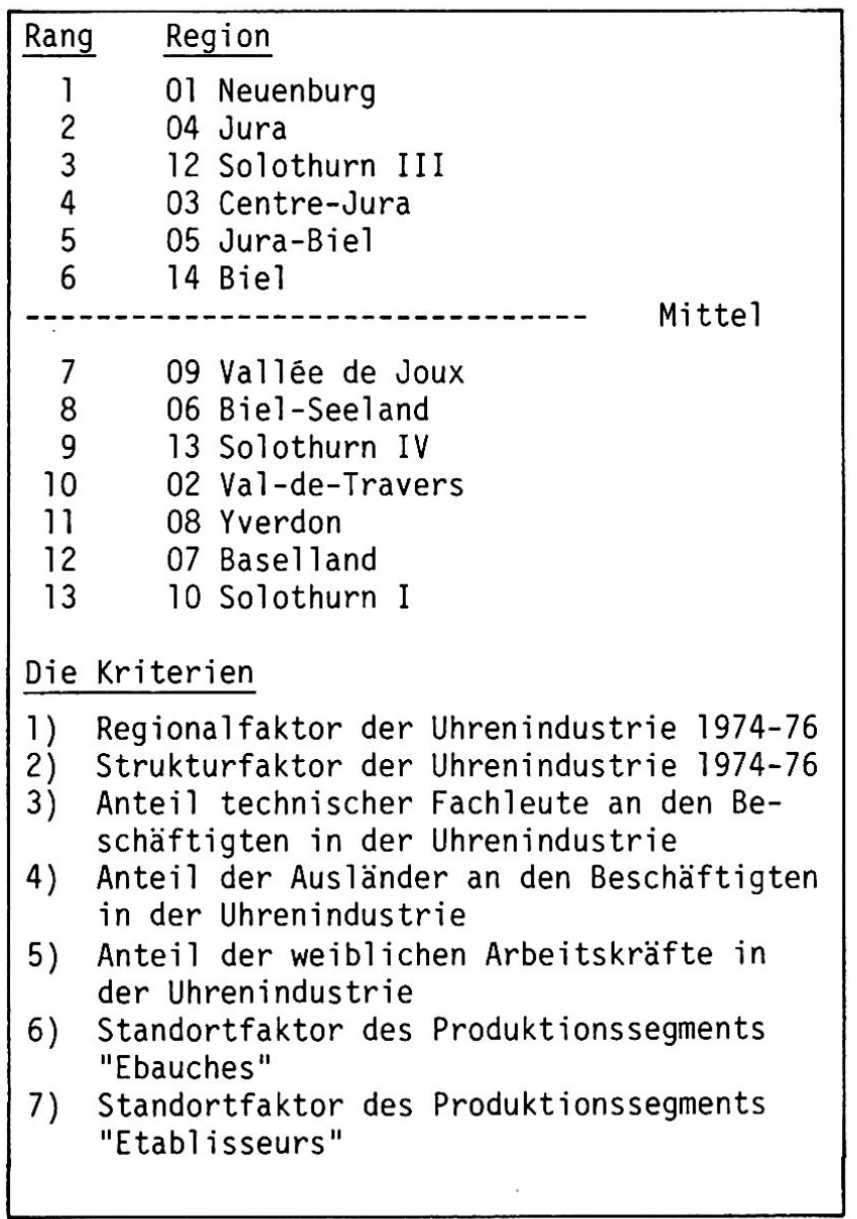

Tab. 2: Klassierung der Regionen nach allgemeinen strukturellen Kriterien 1975

\begin{tabular}{|c|c|}
\hline Rang & Region \\
\hline 1 & 06 Biel-Seeland \\
\hline 2 & 08 Yverdon \\
\hline 3 & 13 Soleure IV \\
\hline 4 & 07 Baselland \\
\hline 5 & 01 Neuenburg \\
\hline 6 & 10 Solothurn I \\
\hline 7 & 05 Jura-Biel \\
\hline 8 & 14 Biel \\
\hline--- & - Mittel \\
\hline 9 & $02 \mathrm{Val}$-de-Travers \\
\hline 10 & 04 Jura \\
\hline 11 & 12 Solothurn III \\
\hline 12 & 03 Centre-Jura \\
\hline 13 & 09 Vallēe de Joux \\
\hline \multicolumn{2}{|c|}{ Die Kriterien } \\
\hline 1) & \multirow{6}{*}{$\begin{array}{l}\text { Standortfaktor der Uhrenindustrie } \\
\text { Standortfaktor der Maschinenbranche } \\
\text { Standortfaktor von Metallindustrie und } \\
\text {-gewerbe } \\
\text { Gesamter Regionalfaktor } \\
\text { Gesamter Strukturfaktor } \\
\text { Bedeutung des Tertiärsektors am Total } \\
\text { der Beschäftigten }\end{array}$} \\
\hline 2) & \\
\hline 3) & \\
\hline 4) & \\
\hline 5) & \\
\hline 6) & \\
\hline
\end{tabular}

Diese Zusammenhänge begründen denn auch die erheblichen Unterschiede in der strukturellen Gefährdung der einzelnen Uhrenregionen.

Die Tabellen 1 und 2 vermitteln die Resultate eines Klassierungsversuchs von D. MAILLAT/J.L. JUVET/B. KUNZ (7) aufgrund von 7 direkt auf die Uhrenindustrie bezogenen Kriterien (Tab. 1) und von 6 allgemeineren strukturellen Indikatoren (Tab. 2) (8).

Ein Zusammenzug dieser beiden Ranglisten ist weder methodisch eindeutig lösbar noch vom politischen Bedarf her notwendig: als weit wichtiger erweist sich die Auffächerung dieser Problemsicht auf die einzelnen Kriterien, kann doch erst aufgrund der Detailanalyse auf denkbare Massnahmenbereiche geschlossen werden. Die Tabellen illustrieren eindruicklich, wie gefährlich Beurteilungen aufgrund von Durchschnittswerten sein können, wenn sie nicht von einer verfeinernden Problemsicht begleitet sird. Zwar sind die Uhrenregionen insgesamt strukturell gefährdet, zwar steht die Uhrenindustrie vor ungelösten Problemen - aber von gleicher Bedeutung sind die interregionalen Differenzierungen, die Unterschiede nach Produktionslinien und die betriebsindividuell keineswegs einheitlichen Verhaltensweisen. Mit welchen bestehenden Massnahmen können nun die Gemeinwesen - Gemeinden, Regionen, Kanton und Bund - auf diese sich aller Voraussicht nach weiter verschärfende Problemlage reagieren? 
ERFOLGSWAHRSCHEINLICHKEIT TRADITIONELLER MASSNAHMEN DER REGIONALPOLITIK

Infrastrukturhilfe für Regionen mit genügender Ausstattung?

Das regionalpolitische Instrumentarium des Bundes und der meisten Kantone liegt sachlich schwergewichtig im Infrastrukturbereich und ist räumlich auf Bergregionen beschränkt (9). Nun fallen die meisten Uhrenregionen unter den räumlichen Geltungsbereich des Investitionshilfegesetzes (IHG) des Bundes; aufgrund detaillierter regionaler Entwicklungskonzepte kann somit der Ausbau ihrer Infrastruktur finanziell tatkräftig unterstützt werden (10).

Mit Blick auf die Uhrenregionen fragt sich nun allerdings, ob diese $\mathrm{Hilfe}$ am richtigen ort ansetzt. Konkret gefragt: bestehen in diesen Regionen genügend notwendige Infrastrukturvorhaben, um spürbare regionalwirtschaftliche Effek te auslösen zu können?

Auch wenn eine sichere Antwort erst aufgrund der noch fertigzustellenden regionalen Entwicklungskonzepte möglich sein wird, kann doch mit $\mathrm{Hilfe}$ vorliegender Schätzungen auf nurmehr wenige notwendige Infrastrukturinvestitionen geschlossen werden (11).

Hinzu kommt, dass die Infrastrukturpolitik ganz grundsätzlich unter den neuen Rahmenbedingungen weitgehend stagnierenden Wirtschafts- und Bevölkerungswachstums an Bedeutung für die Regionalpolitik zu verlieren scheint. Sie beruht ja grundsätzlich auf der Annahme, dass über die Gestaltung der regionalen Ausstattungsstruktur der Mobilitätsgrad und v.a. die Mobilitätsrichtung von Bevölkerung und Unternehmen in wesentlichem Ausmass beeinflusst werden kann. Nun ist zu vermuten, dass mit reduziertem Wachstum auch ein wesentlich reduziertes Mobilitätspotential und wahrscheinlich auch eine eingeschränkte Mobilitätsbereitschaft verbunden sind (12). Die im Dienste der Regionalpolitik stehende Infrastrukturpolitik ist daher ganz grundsätzlich in ihrer Wirkung in Frage gestellt, ganz besonders natiurlich in Regionen mit bereits recht gut entwickelter Ausstattung.

\section{Zögernder Ausbau der flankierenden Massnahmen}

Direkt an das IHG und an das Bestehen regionaler Entwicklungskonzepte gebunden sind zwei flankierende Massnahmen ausserhalb des Infrastrukturbereiches (13):

- Die Bürgschaftsgewährung für Klein- und Mittelbetriebe im Berggebiet, welche eine Verpflichtung des Bundes bis zu einer zu verbürgenden Hauptschuld von Fr. 50'000.- erlaubt;

- die Regelungen im revidierten Hotel- und Kurortkreditgesetz, welches für touristische Anlagen, für Renovationen und für den Neubau von Hotels und Personalunterkünften Finanzhilfe in Form von zinsgünstigen, langfristigen Darlehen und von Bürgschaften vorsieht.

Beide Massnahmen decken einen offensichtlich grossen Bedarf vorab von Klein- und Mittelbe- trieben in strukturschwachen Räumen ab. Ihre Stosskraft ist jedoch in institutioneller und finanzieller Hinsicht recht schwach - auf jeden Fall mit Blick auf den Problemdruck in den Uhrenregionen eindeutig ungenügend.

Weitere regionalpolitische Bemühungen des Bundes sind ebenfalls auf die Berggebiete beschränkt, so insbesondere agrar- und forstwirtschaftliche Massnahmen (14) und Wohnbau- und -renovationshilfen (15). Eine neuartige Idee, nämlich die Förderung des Ausbaus von Ferienwohnungen in bestehender Wohnsubstanz im Berggebiet, ist konkret vorbereitet, im Zeichen einer Politik des Budgetausgleichs jedoch vorläufig sistiert worden (16).

\section{Fazit: grosser Problemdruck und kleines Echo}

Vereinfachend kann somit gefolgert werden, dass zumindest auf Bundesebene praktisch keine Massnahmen zur Verminderung der beschriebenen regionalpolitischen Problematik bereitstehen. Einige Uhrenkantone versuchen allerdings, eine Antwort zu geben, v.a. die Kantone Bern, Solothurn und Neuenburg (17). Die bisher realisierten Massnahmen sind im Ansatz ähnlich, auch wenn signifikante Unterschiede institutioneller und v.a. finanzieller Art bestehen. Auf die Charakteristik dieser regional- und strukturpolitischen Vorkehren wird zurückzukommen sein; vorweg muss ihre gesamte Wirkungsstärke trotz einzelnen Erfolgsmeldungen jedoch als bescheiden eingestuft werden, dies v.a. aufgrund ihrer ungenügenden finanziellen Tragkraft. In dieser Situation wird das Ueberdenken traditioneller regionalpolitischer Strategien und die Ueberprüfung neuartiger Ansätze zur Notwendigkeit und zur Voraussetzung für mehr Innovation in der Politik (18).

THEORETISCHER ANSATZ: INNOVATIONEN ALS TREIBKRAEFTE REGIONALWIRTSCHAFTLICHER ENTWICKLUNG

\section{Strukturprobleme als Ausgangspunkt}

Die Probleme der Uhrenindustrie - und damit der Uhrenregionen - lassen sich auf konjunkturelle (z.B. Rückgang der Nachfrage in gewissen Produktesegmenten) und auf strukturelle Ursachen (z.B. Defizite in der Forschung und Entwicklung) zurückführen. Diese Ursachen sind eng miteinander verflochten, ihr einzelweises relatives Gewicht ist kaum eruierbar. In unserem Zusammenhang sind jedoch v.a. jene Faktoren von Bedeutung, die durch die eidgenössische oder kantonale Wirtschaftspolitik wesentlich beeinflusst werden können. Damit konzentriert sich die folgende Betrachtung folgerichtig auf strukturelle Ursachen der Problematik, da sowohl die Weltkonjunktur, die Entwicklung neuer Zollpräferenzen (z.B. Erziehungszölle für die Uhrenindustrie in gewissen Ländern) als auch das Währungsproblem als wichtigste externe Faktoren durch die schweizerische Wirtschaftspolitik nicht oder zumindest nur marginal beeinflussbar sind.

Parallel zu jeder wirtschaftlichen Entwicklung verändern sich laufend mikro- und makroökonomi- 
sche Strukturen; daraus entstehende Strukturprobleme regionaler, sektoraler oder betriebsindividueller Art sind nichts Neues (19). Sie lassen sich in aller Regel auf entsprechende Anpassungsprobleme einzelner Firmen oder ganzer Branchen (die - wie die Uhrenindustrie allenfalls sogar regional konzentriert sind) an veränderte Marktbedingungen zurückführen. Im Falle der schweizerischen Uhrenindustrie bedeutet dies vorab:

- Anpassungsiücken im Bereich von Forschung und Entwicklung

- zu zersplitterte Branchenstruktur bzw. ungenügende Konzentration der unternehmerischen Kräfte in Produktion und Handel

- Defizite im Distributionsbereich, insbesondere im Bereich der Werbestrategie und im Aufbau von Distributionsnetzen.

Diese (und weitere) strukturpolitischen Ursachen haben zum beschriebenen Arbeitsplatzverlust wesentlich beigetragen und in der räumlichen Konzentration des Verlustes auch eine regionalpolitische Dimension geschaffen. Diese wird erst dann zur schweizerischen Problematik, wenn sie auf die bestehenden strukturpolitischen Ziele des Gemeinwesens bezogen wird; die beiden wichtigsten lauten dabei:

- Erhaltung der Wettbewerbsfähigkeit der schweizerischen Volkswirtschaft (20) - Abbau regionaler Ungleichgewichte (21) Beide Ziele erhalten unter der Rahmenbedingung verhaltenen wirtschaftlichen Wachstums zunehmend Gewicht, da offensichtlich in dieser Phase Anpassungsprozesse schwieriger ablaufen und entsprechende strukturelle Probleme weniger leicht lösbar sind als in Zeiten starken Wirtschaftswachstums (22).

Strukturelle Probleme lassen sich somit in aller Regel auf Defizite in der Fähigkeit zur Erkennung von und Anpassung an veränderte Marktdaten zurückführen. Dafür können verschiedene Gründe massgebend sein; im Falle der Uhrenindustrie spielten u.a. eine jahrelange "Position der Stärke" auf den Weltmärkten nicht zuletzt aufgrund staatlicher Hilfe (Uhrenstatut) sowie ein offensichtlich lange Zeit unterbewerteter Schweizerfranken eine gewichtige Rolle. In unserem Zusammenhang sind diese Anpassungsschwäche und diese Innovationsarmut für die gegenwärtige Problematik von entscheidender Bedeutung.

\section{Was sind Innovationen?}

Im Grossen Brockhaus von 1954 wird das Wort "Innovation" überhaupt noch nicht erwähnt. Im Duden des gleichen Jahres wird Innovation mit "Verjüngungvorgang mancher Pflanzen" umschrieben. So unwichtig war der Terminus vor gut 20 Jahren.

Unterdessen hat er sich zum Schlagwort entwikkelt. Mit der Häufigkeit des Gebrauches ist auch das Bemuihen um Definitionen gewachsen. Eine davon lautet:

"Unter Innovation wird jeder Prozess verstanden, der auf die bewusste Herbeiführung einer Aenderung mit gewünschten Eigenschaften abzielt" (23).
Zwei Eigenschaftswörter sind dabei besonders interessant, nämlich "bewusst" und "abzielt". Das bedeutet Ziel und Planung. "Innovation ist ein Vorgang, der durch Planung ökonomisch sinnvoll auf ein festes Ziel hin ausgerichtet werden kann" (24).

Innovationen vollziehen sich dauernd in Oekonomie, Technik, Wissenschaft, Kunst, Politik - wenn auch in den einzelnen Zeitphasen unterschiedlich intensiv.

Für den Bereich der Wirtschafts- und Strukturpolitik ist eine weitere Konkretisierung des Begriffes unerlässlich und auch möglich: unter Innovation wird hier die Einführung neuer Produkte, neuer Techniken, neuer Ressourcen und Materialien, neuer Organisationsformen und neuer Absatzmärkte verstanden; "neu" ist dabei naturgemäss relativ zu verstehen, entwickeln sich diese Neuheiten doch meist aus Bestehendem. Immerhin wird unter Innovation ein signifikanter Schritt in weiterentwickelte und neue Gebiete verstanden. In dieser "Kunst des Andersmachens" sah bereits SCHUMPETER vor einigen Jahrzehnten die treibende Kraft der wirtschaftlichen Entwicklung.

Der Ansatz hat sich zum zentralen Ausgangspunkt der modernen Wirtschaftspolitik entwickelt. Er besitzt auch eine kräftige ordnungspolitische Komponente: in einem marktwirtschaftlichen System gleicht der Wettbewerb zwischen Unternehmern einer Art Entdeckungsverfahren, in dem neue Produktionsverfahren, neue Produkte, neue Märkte, neue Standorte u.w.m. erkundet werden. "Unternehmer dienen gleichsam als Kundschafter in dem Neuland der künftigen Produktionsstrukturen. Sie wagen einen Einsatz. Verlieren sie und machen Verluste, so weiss man, dass der von ihnen eingeschlagene Weg nicht gangbar ist. Andere werden gewinnen. Für die Gesamtwirtschaft ist es wichtig, dass in diesem Spiel genug Einsätze gewagt werden" (25).

Genau hier liegt gegenwärtig denn auch das Problem: Rezession und ausserordentlich rascher Strukturwandel haben eine tiefe Verunsicherung der Investoren verursacht. Unsicherheit in den Preisen, Techniken, Märkten, Produkt-Wettbewerbsfähigkeiten und Währungsrelationen führen zu kleinen, ungenügenden Einsätzen.

Hier setzen denn auch die neuern wirtschaftspolitischen Massnahmen der industrialisierten Staaten - auch der Schweiz (26) - ein: in der Schaffung von Anreizen für Investitionen in Innovationen. Darunter fallen Finanzhilfen aller Art für Forschung und Entwicklung, Sonderbeiträge für Erstinnovationen oder Steuererleichterungen zur Induzierung von "Pioniergewinnen" u.a.m. (27). Entscheidend ist in unserem Zusammenhang nicht die genaue Art des Einsatzes staatlicher Hilfen, vielmehr der weitgehend einheitliche Ansatz. Innovationsschwäche ist mit Sicherheit der wichtigste langfristige Engpassfaktor für die wirtschaftliche Zukunft hochentwickelter Länder. Die durchschnittlichen Aufwendungen für neue Innovationen steigen rasch an. Der Erneuerungsdruck wächst. Die Entwicklungszeit für neue Produkte steigt, ihre durchschnittliche Lebensdauer sinkt. 
Höheren durchschnittlichen Kapitalkosten stehen somit kleinere und zeitlich kürzere Gewinnerwartungen gegenüber. In der Folge wächst der Abschreibungsdruck, er verursacht direkt Innovationsbarrieren. In diesem Zusammenhang lässt sich eine subsidiäre staatliche Hilfe wohl rechtfertigen (28).

\section{Disparitäten in der Innovationsfähigkeit als} regionalpolitisches Grundproblem

Konzentriert sich der Engpassfaktor "Innovationsfähigkeit" nun in gewissen Teilräumen, so verursacht er direkt und innert kurzer Zeit tiefgreifende und langfristige regionalpolitische Probleme. Die regionalpolitische Dimension des theoretischen Ansatzes ergibt sich somit aus räumlichen Disparitäten in der Innovationsintensität der betreffenden Unternehmungen.

Regionale Innovationsschwäche kann vorab auf zwei Ursachen zurückgeführt werden:

- Einerseits kann eine durch Innovationsschwäche gekennzeichnete Branche räumlich konzentriert angesiedelt sein. Das dargestellte Beispiel der Uhrenindustrie illustriert deutlich die direkten und verhängnisvollen Auswirkungen einer solchen weitgehenden Monostruktur auf die gesamte regionale Wirtschaft.

- Anderseits können die Aufnahme- und Verwertungseigenschaften für neue Ideen in gewissen Regionen unterdurchschnittlich entwickelt sein. Der Engpassfaktor liegt dann nicht direkt in der geringen Innovationsintensität der betreffenden Branchenstruktur, sondern in der Innovationsschwäche der anwesenden Betriebe. Im Extremfall kann in einer Region optimaler Branchenstruktur Innovationsarmut bestehen, weil die einzelnen Unternehmen entweder deutlich unter dem betreffenden Branchenniveau liegen oder weil sie keine Entscheidungsmacht mehr besitzen. Diese zweite Erklärung besitzt wahrscheinlich für alle Peripherräume wachsende Bedeutung, ist doch eine rasch zunehmende Konzentration einzelbetrieblicher Entscheidungsmacht auf die wichtigsten Wirtschaftszentren zu beobachten. Dieser Wandel führt in aller Regel zur Abwanderung betriebswirtschaftlicher Headquarter-Funktionen aus den Peripherräumen und damit zu abnehmenden Aktivitäten vorab in den Bereichen Forschung und Entwicklung, Planung, Marketing, Finanzen und Organisation (29). Für die betreffenden Regionen resultiert daraus ein Verlust an funktionaler Eigendynamik und damit eine erhebliche Innovationsschwäche in den einzelnen Betrieben.

\section{Einzelbetriebliche Innovationsschwächen}

Die bisherigen Ausführungen haben für das Beispiel der Uhrenindustrie die erste Erklärungsmöglichkeit für regionale Strukturschwäche den branchenstrukturellen Ansatz - deutlich belegt. Dabei konnte nachgewiesen werden, dass auch bei einer relativ klar abgegrenzten Bran- che signifikante Unterschiede zwischen den einzelnen Produktelinien bestehen.

Der zweite Erklärungsansatz treibt nun diese Differenzierung weiter auf die einzelbetriebliche Ebene: auch innerhalb der einzelnen Produktelinien müssen offensichtlich erhebliche Unterschiede in der Innovationsfähigkeit von Betrieb zu Betrieb bestehen. Sie sind zurückzuführen auf unterdurchschnittliche Anpassungsfähigkeiten oder/und auf Defizite an Headquarter-Funktionen. Auch für diese Erklärungsmöglichkeit sind empirische Belege für die Uhrenregionen zumindest ansatzweise möglich; zwei Beobachtungen seien näher ausgeführt:

a) Die schweizerische Uhrenbranche ist im Gegensatz zu ihren wichtigsten Konkurrenten ( $v . a$. Japan und USA) nur durch wenige vertikal organisierte Grosskonzerne und als Ganzes durch eine ausgeprägte horizontale Struktur charakterisiert. Ueber 600 hochspezialisierte Betriebe produzieren Komponenten des Werks und des Habillements, etwa 350 Etablisseure konzentrieren sich auf die Erstellung der Kollektion, das Zusammensetzen der Uhr und ihren Vertrieb. Diese Spezialisierung und die damit verbundene ausgeprägt kleinbetriebliche Struktur ist offensichtlich dem rasch gewachsenen und in erster Linie auf die Einführung der Elektronik zurückzuführenden internationalen Erneuerungsdruck nicht gewachsen:

- Die einzelnen Betriebe haben lange Zeit nur ihren Teilmarkt gesehen und nur an ihre Spezialität gedacht. Neue Marktdaten haben sie überrascht. "Der Handlungsspielraum der einzelnen Firmen ist eng begrenzt" (30).

- Die Abstimmung der Produkteplanung von der Forschungs- und Entwicklungsphase bis zur Marktreife ist in erheblichem Masse erschwert: die Entwicklung und Einführung neuer Produkte braucht mehr (kostbare) Zeit als in vertikal durchorganisierten Konzernen.

- Horizontale Struktur bedeutet auch dauerndes einzelbetriebliches Bemuihen um Abgrenzung gegenüber dem Nachbarn. Die schweizerische Uhrenindustrie kennt beispielsweise mehrere Hundert Werkskaliber. Das Sortiment ist entsprechend breit, was $z u$ kleinen Produktionsserien, zu hohen Durchschnittskosten ab Fabrik und damit zu schmalen Margen führt (31).

- Ausrichtung auf bestimmte und meist enge Produktsegmente, Beharren auf Marktnischen, verlängerte Entwicklungszeiten für neue Produkte und die Erwartung nur kleiner Seriengrössen führen gemeinsam zu einer Lähmung des allenfalls noch vorhandenen Innovationswillens. Ein zu langes Verharren auf traditionellen Produkten und Techniken schafft kaum mehr zu überwindende Innovationsbarrieren, ist doch die Entwicklung aus dem Bisherigen, das "Bewahren durch Verändern" (32) darum in Frage gestellt, weil der für einen Anschluss an die technologische Weltspitze notwendige Innovationsschritt zu gross und zu teuer wird. Er ist nur noch Unternehmen mit kräftiger techno- 
logischer Eigendynamik und hoher Kapitalausstattung möglich. Die Multivarianz der übrigen Betriebe tendiert gegen null (33).

Nachgewiesen ist somit, dass sich die betriebliche Innovationsfähigkeit auch aus der spezifischen Situation des Betriebes innerhalb der betreffenden Branche und aus seinen Management-Qualitäten ergibt. Mit diesen Faktoren lassen sich in aller Regel betriebliche Unterschiede bezüglich der Innovationsfähigkeit innerhalb einer Produktelinie erklären.

b) Die Abwanderung betriebswirtschaftlicher Entscheidungsfunktionen aus den Uhrenregionen in wirtschaftliche Zentren im Mittelland ist bisher nicht systematisch untersucht worden (34). Unklarheit herrscht allerdings lediglich über die Intensität dieses Wandels, nicht jedoch uiber sein Bestehen. Zu deutlich blinken entsprechende Warnsignale auf; zu erinnern ist beispielsweise an die Verlagerung von Headquarter-Funktionen der zweitgrössten Uhrenholding (SSIH) nach Zürich (35) oder an entsprechende Standortentscheide dynamischer Mittelbetriebe (36).

Bedeutenden Einfluss auf diesen räumlichen Wandel üben neue technologische Innovationen aus, im Falle der Uhrenindustrie also die Elektronik. Die Herstellung elektronischer Bestandteile für eine Uhr ist keineswegs mehr an traditionelle Standorte gebunden. So ist auch erklärbar, warum grundlegende Anstrengungen im Bereich von Forschung und Entwicklung, aber auch in der Produktion in den Agglomerationen Zürich (z.B. FASELEC, Brown Boveri) und Basel (z.B. Hoffmann-La Roche) realisiert werden. Die mehrdimensionale Anwendbarkeit solcher elektronischer Elemente würde an sich der Uhrenbranche Gelgegenheit zur Diversifikation in andere Produktefelder (z.B. in der Apparateindustrie) bieten. Umgekehrt besteht für dynamische und technisch hochentwickelte Betriebe aus andern Branchen die Chance, diese Elemente für die Uhrenindustrie zu entwickeln und zu produzieren.

Es scheint, dass die zweite Chance besser genutzt und damit der beschriebene räumliche Konzentrationsprozess als Folge betriebswirtschaftlicher Entscheidungen beschleunigt wird.

MERKPUNKTE FUER EINE INNOVATIONSORIENTIERTE REGIONALPOL ITIK

Die beschriebenen, neuartigen strukturellen und regionalen Probleme führen mit Blick auf die beiden genannten strukturpolitischen Zielsetzungen direkt zu markanten Ecksteinen eines zumindest für die Schweiz bisher weitgehend unberührten regionalpolitischen Aktionsfeldes. Innovationsschwäche als grundlegende Ursache regionalwirtschaftlichen Abschwungs kann offensichtlich mit den traditionellen Massnahmen nicht behoben werden. Eine grundsätzliche strategische Neuorientierung in Richtung einer innovationsfördernden Regionalpolitik erscheint als Antwort auf die umschriebene Problematik not- wendig, auf alle Fälle zielkonform und daher zumindest diskussionswürdig.

Aus der vorstehenden Analyse lassen sich für eine solche Strategie vorerst drei übergeordnete Grund. sätze ableiten:

1. Alles Handeln und Verhalten der Gemeinwesen mit erheblichen strukturellen Effekten hat sich auf die beiden genannten übergeordneten Zielsetzungen zu beziehen. Konkret bedeutet dies eine deutliche Absage an strukturerhaltende Interventionen, beispielsweise also an die Idee eines Bundeslazaretts für kranke Betriebe (37). Erlaubt wären somit nach diesem Grund. satz allein Massnahmen, welche die Anpassung an veränderte Strukturdaten erleichtern bzw. fördern.

2. Die Motivation für eine innovationsorientierte regionalpolitische Strategie ergibt sich v.a. aus der Tatsache räumlicher Konzentration von Innovationsschwäche bestehender Betriebe. Denk. bar wäre nun auch eine Problemlösung durch Implantierung neuer und zukunftsträchtiger Betriebe in den betreffenden Regionen, also eine Strategie der regionalen Diversifizierung. Angesprochen sind damit v.a. zahlreiche neuere Massnahmen der kantonalen Wirtschaftspolitik zur Begünstigung der regional gesteuerten Industrieansiedlung (38). Auch wenn diese Politik Teilerfolge buchen kann, wird sie zunehmend aus zwei Gründen in Frage gestellt: einerseits besteht bereits heute und gerade zwischen den Uhrenkantonen eine erhebliche interkantonale Konkurrenz; ein gegenseitiges "Aufwiegeln" durch immer attraktivere wirtschaftspolitische Massnahmen kann innert kurzer Zeit für die betreffenden Kantone kontraproduktive Effekte auslösen. Anderseits ist auf den ausserordentlich starken Rückgang an Mobilität und Mobilitätsbereitschaft von Unter. nehmen unter den gegenwärtigen Rahmenbedingungen hingewiesen worden. Die neue Strategie stellt daher den mobilitätsorientierten Ansatz zumindest teilweise in Frage und konzentriert sich im Gegensatz dazu vorab auf das bestehende regionalwirtschaftliche Potential.

3. Dieses Potential ist keineswegs einheitlich weder bezüglich Branchen noch bezüglich Produktelinien noch bezüglich Betriebsgrössen. Es ist im Gegenteil charakterisiert durch grosse betriebsindividuelle Unterschiede, die sich zu entsprechenden regionalen Differenzierungen summieren können.

Daher muss jede auf Sektoren, Branchen, Produktelinien oder Betriebsgrössen ausgerichtete Strukturpolitik als höchst problematisch beurteilt werden. Was möglich bleibt, ist eine regional und einzelbetrieblich konzipierte innovationsfördernde Strukturpolitik (39).

Durch diese drei Merkpunkte ist ein strukturpolitischer Bereich abgesteckt, der ganz bestimmte und zielbewusste Aktivitäten der öffentlichen Hand zulässt. Vorab können vier Aktionsbereiche direkter und indirekter Einflussnahme (ebenfalls im Sinne von Merkpunkten) genannt werden: 
4. Die staatlichen Hilfen - seien dies nun Steuererleichterungen, direkte oder indirekte $\mathrm{Fi}$ nanzierungsbeihilfen - sind auf innovatorische Tätigkeiten einzelner Betriebe in strukturschwachen Räumen auszurichten (40). Anzustreben ist die Erhöhung der Innovationsfähigkeit und -diffusion. Notwendige Voraussetzung dafür ist die Förderung von anwendungsorientierter Forschung und Entwicklung (41).

5. Der regionalpolitische Ansatz führt zur Forderung nach räumlich konzentriertem Einsatz dieser Mittel. Nur so lassen sich wichtige kritische Schwellenwerte überschreiten. Nur so ist auch eine "innovationsträchtige Atmosphäre" induzierbar, die entsprechende. Fühlungsvorteile schafft und neue Diversifikations- und Kooperationsmöglichkeiten aufdeckt. Entscheidende Merkmale der Strategie mussen darum "Masse" und "Kontinuität" sein (42).

6. Die Erfolgswahrscheinlichkeit dieser Massnahmen hängt aber auch von ihren Adressaten ab. Die Uhrenindustrie ist durch eine horizontale und nach wie vor starre Struktur charakterisiert. Diese ungünstigen Vorzeichen müssen sich wandeln, die zweifelsohne bestehenden brancheninternen Identifikationsprobleme (43) müssen gelöst werden. Es ist immerhin denkbar, dass die Massnahmen der öffentlichen Hand entsprechende organisatorische und brancheninterne Veränderungen beschleunigen helfen.

7. Gerade dieses Ziel der möglichst grossen Effizienz staatlichen Mitteleinsatzes könnte auch dadurch angenähert werden, indem Kooperations- und Koordinationsaktivitäten in den Bereichen von Forschung und Entwicklung, Innovationen und Diversifikationen honoriert würden - sei dies durch ein Bonussystem oder durch entsprechende projektbezogene Steuerermässigungen. Ohne Zweifel könnten so erhebliche Rationalisierungsreserven ausgeschöpft und optimalere $\mathrm{Zu}$ - und Ablieferungsbeziehungen entwickelt werden.

\section{FORSCHUNGSLUECKEN ALS VORBEHALT}

Die Entwicklung irgendeiner neuen politischen Strategie benötigt eine Reihe von Entscheidungsgrundlagen. Erst durch Wissen entsteht Entscheidungsmacht. Diese These stimmt auch dann, wenn man sie als Gleichung auffasst und mit minus eins multipliziert: Unwissen ist Ohnmacht. Das Wissen, auf das es in unserem Zusammenhang ankommt, liegt erst ansatzweise vor. Erhebliche Forschungslücken bestehen vorab in folgenden Bereichen:

- Bereits die branchenstrukturellen Aussagen basieren in der Schweiz auf einer schwachen statistischen Grundlage. Diese Grundlage wird zur dünnen und brüchigen Eisdecke, wenn es um betriebsindividuelle Strukturdaten geht. Beim beschriebenen betriebsbezogenen Ansatz werden jedoch gerade solche Angaben aus dem "Intimbereich" der Unternehmen benötigt. Zudem ist unklar, welche Kombination von Indikatoren notwendig und hinreichend ist und auf welche Wei- se sie verfügbar gemacht werden kann.

- Die gewünschte Datenkombination hängt nicht zuletzt davon ab, mit $\mathrm{Hilfe}$ welcher Indikatoren die Innovationsintensität eines Betriebes bzw. eines Projektes beurteilt werden soll. Anzustreben wäre die präzise Erfassung innovatorischer Engpassindikatoren. Ueber deren Charakteristik und Gewicht bestehen praktisch keine empirischen Untersuchungen.

- Diese Diskussion über die Erhebung von Engpassfaktoren muss in eine eingehende und v.a. empirische Untersuchung über die Determinanten und Mechanismen von Innovations- und Diffusionsprozessen eingebettet sein. Verschiedene theoretische Determinantensysteme bestehen bereits (44). Ungeklärt ist ihra empirische Standfestigkeit, die erst Aufschluss über die vergleichsweise Bedeutung einzelner Faktoren zu geben vermöchte.

- Die Diffusion von Innovationen ist v.a. in ihrer räumlichen Dimension ungeklärt. Der Verlauf von Know-how-Transfers beispielsweise von der Agglomeration in Richtung Peripherräume ist kaum erforscht, daran gebundene Probleme vorab im Kommunikationsbereich harren einer Lösung.

- Die Intensität und Geschwindigkeit dieser Transfers von Wissen hangen auch von der Aufnahmefähigkeit und Erwartungsbereitschaft der "Kunden" von Neuerungen ab. Grundsätzlich nachgewiesen ist für diese "Nachfragequalitäten" eine grosse Bedeutung der erwähnten Headquarter-Funktionen. Unbekannt ist hingegen die einzelweise Rolle dieser Funktionen im Transfer-Prozess sowie das Ausmass des räumlichen Konzentrationsprozesses dieser wichtigen betriebswirtschaftlichen Funktionen.

Weitere Forschungslücken sind zweifelsohne vorhanden. Sie sind derart gross, dass sie sich zu einem Vorbehalt gegenüber einer innovationsorientierten Regionalpolitik summieren. Es ist immerhin denkbar, dass verbesserte Entscheidungsgrundlagen aufgrund wissenschaftlicher Erkenntnisse das harte strukturpolitische Klima (45) mildern würden. Voraussetzung einer innovationsorientierten Regionalpolitik durfte somit eine Phase innovationsfreudiger Forschung sein.

\section{Anmerkungen}

1) Detaillierte Aufschlüsse vermjttelt die Studie "L'emploi dans la région horlogère", Teil II, von D. Maillat/J.L. Juvet/B. Kunz; Neuenburg, März 1978

2) Ausdruck dafür ist beispielsweise ein Zulieferantenverzeichnis für den Zentral-Jura, erstellt von der RET SA, La Chaux-de-Fonds (1976)

3) Ausführlich werden diese Ursachen und Mechanismen dargestellt in der Studie "Die Wettbewerbsstellung der schweizerischen Uhrenindustrie" von Wilhelm Hill, Bern 1977 und in der brancheninternen Untersuchung " $L$ 'horlogerie suisse dans la vision des 10 ans à venir", erstellt im Jahre 1976 durch eine Expertengruppe im Auftrag der Fédération horlogère suisse

4) Nicht nur bestehen gravierende Unterschiede 
zwischen elektronischen und mechanischen Produkten, sondern auch zwischen den einzelnen Gliedern der einzelnen Produktionsketten. So ist bei den Termineurs, Assortiments, Balanciers, Ressorts, Cadrans mit weiteren deutlichen Arbeitsplatzverlusten zu rechnen, während beispielsweise die Ebauches-Fabrikation eher gute Marktaussichten hat. Vgl. zur weiteren Differenzierung Maillat/Juvet/Kunz a. a.O.III. Seite 2 ff.

5) So sind insbesondere folgende Regionen mit eindeutigen Produkteschwerpunkten ausgezeich net (Zahlen 1976 nach Arbeitsplatzanteilen) Centre Jura: 28,6\% aller Terminers; $17 \%$ der Boites-Herstellung

Soleure III: 47,8\% der Ebauches-Fabrikation Neuenburg: $\quad 34,0 \%$ der Ebauches-Fabrikation Jura: $\quad 53,4 \%$ der Boites-Herstellung Je nach Marktaussichten der betr. Produktelinie sind diese Regionen durch einen unterschiedlichen Grad an Strukturschwäche gekennzeichnet. Da zumindest seit 1974 diese Marktdaten ständig ändern, sind alle derart monostrukturierten Räume potentiell strukturell gefährdet.

6) Diesbezügliche eigene Untersuchungen mittels Interviews zeigen ausserordentlich grosse Unterschiede in der Fähigkeit des Managements zur richtigen (und ehrlichen) Problembeurteilung, zur Zielfindung und zur Diskussion von Massnahmen. Die grössten Unterschiede sind allerdings in der Phase der Realisierung und Durchsetzung von Massnahmen festzustellen. Vgl. dazu im Sinne eines "Querschnitts": DER BUND vom 25.4.1976, Seite 95; DER BUND vom 4.6. 1977 Seite.37; Tribune-Le Matin vom 21. 3. 1978 Seite 5; NZZ Nr. 73 vom 30.3. 1978 Seite 15

7) Maillat/Juvet/Kunz a.a.O.,III. Seite 28. Die einzelweise Interpretation der in Tab. $1+2$ angegebenen Indikatoren kann aus Platzgründen nicht wiedergegeben werden.

8) Dieser Versuch ist auch methodisch interessant, da er auf die empirische Realisierbarkeit aug 20 gerichtet ist. Nicht zuletzt darum steht er in deutlichem Gegensatz zu der in der Absicht gleichgelagerten, aber theoretisch ausgerichteten Studie "Grundsätzliche und methodische Aspekte der Frühindikation von Problemen der Branchenstruktur"; Bericht zuhanden des Eidg. Volkswirtschaftsdepartementes der Eidg. Studienkommission für Preis-, Kosten- und Strukturfragen, Bern 1977

9) Zum regionalpolitischen Instrumentarium in der Schweiz vgl. Mühlemann Fritz: Von der Berggebietsförderung zur regionalen Entwicklungspolitik, in: Wirtschaftspolitische Mitteilungen $7 / 8 \quad 1977$

10) Die Details dieser Hilfen sind beispielsweise umschrieben in Simon Huber, Der Stand der regionalen wirtschaftsförderung des Bündés, in: Informationsbulletin FVV $3 / 1976$ (Bern)

11) Ansätze dazu finden sich in: Michel Rey, Région Jura - quel intérêt pour les communes? Biel, 1977 Daniel Liechti, Vers une nouvelle politique économique de la République et canton Neuchâ- tel, Neuchâtel, 1976

Denis Maillat/J.P. Pellaton, La région CentreJura, Neuchâtel 1975

12) Diese Thesen sollen im Rahmen des Nationalen Forschungsprogrammes "Regionalprobleme in der Schweiz" eingehend überprüft werden.

13) Diese flankierenden Massnahmen werden eingeher dargestellt in Mühlemann Fritz, a.a.o.

14) Dazu beispielsweise Kommission Popp, Ausgleicl zahlungen an die schweizerische Landwirtschaft Bern 1973

15) Niedergelegt im Bundesgesetz über die Verbesserung der Wohnverhältnisse im Berggebiet

16) Der provisorische Gesetzesentwurf trägt den Titel: Kredithilfe an Ferienwohnungen im Berggebiet

17) Dieses Instrumentarium kann in diesem Artikel nicht detailliert umschrieben werden; es sei deshalb hingewiesen auf folgende Literatur: Für eine Uebersicht: Stephan Bieri/Christian Muggli, Massnahmen der kantonalen Wirtschaftspolitik, Aarau 1977

Für Bern: Berggebietsförderung im Kanton Bern, Wirtschaftsbulletin der Kantonalbank von Bern, Nr. 19, Bern 1976

Für Solothurn: Verordnung über Sofortmassnahmen zur Förderung einer regional und strukturell ausgewogenen wirtschaftlichen Entwicklung, Amtsblatt des Kantons Solothurn Nr. 28, 1977

Für Neuenburg siehe Anmerkung II

18) Die folgende Darstellung basiert somit nicht auf der "offenbar urhelvetischen Neigung, das Urteil über das sachlich Mögliche von vornherein auf das politisch Fragbare auszurichten" (Imboden, Helvetisches Malaise, Seite 6, Zürich 1964); angestrebt wird jedoch auch nicht eine Utopie, vielmehr sollen die Vorschläge als Argumente in der gegenwärtigen regionalpolitischen Diskussion dienen.

19) Vgl. dazu beispielsweise die wirtschaftsgeschichtliche Studie von David S.Landes, The Unbound Prometheus, Cambridge 1969

20) F. Kneschaurek, Bedrohte wettbewerbsfähigkeit der Schweiz? In: NZZ Nr. 201 vom 28./29.8.76 S. 17;

H. Kleinewefers, Schwindendes Vertrauen in die Marktregulierungskräfte, in: NZZ vom 16./17. $10.76 \mathrm{~S} .17$

21) F. Mühlemann a.a.o. Seite Iff.

22) Vgl. dazu den aufschlussreichen Artikel von Gerhard Fels (Institut für Weltwirtschaft in Kiel) "Hier irrten die Sachverständigen" in Frankfurter Allgemeine (Serie: "Strategie für magere Jahre", Beitrag XLVI 1976.

23) Die Definition stammt von $H$. Rittel und w.Kung (Berkley/Heidelberg)

24) Zitat aus dem grundlegenden Artikel von Karl Baumgartner in der Frankfurter Allgemeine vom 24.2. 1977 (Serie: "Innovation-Bewahren durch Verändern", Beitrag $I$ )

25) Zitat aus dem Artikel "Wo die deutsche wirtschaft Erfindungsgeist braucht" von ErnstJürgen Horn (Institut für Weltwirtschaft, Kiel) in der Frankfurter Allgemeinen vom 28.2 . 1977 (Serie "Innovation-Bewahren durch Verändern, Beitrag II) 
26) Das vom Delegierten für Konjunkturfragen konzipierte "Impulsprogramm" konzentriert sich auf die Förderung der Anwendung neuer Technologien, der Weitervermittlung von knowhow und der Erschliessung neuer Märkte. Das Programm dürfte frühestens Anfang 1979 in Kraft treten.

27) Vgl. zum diesbezüglich breiten Sortiment an staatlichen Hilfen in der BRD den Artikel von Werner Gries "Milliardenhilfe vom Staat - ein Förderungswegweiser für Forschung und Innovation" in der Frankfurter Allgemeinen vom 18. April 1977 (1. Teil) und 21. April 1977 (2. Teil); (Serie "Innovation-Bewahren durch Verändern", Beiträge XV und XVI)

28) Allerdings gehen alle Arten von staatlichen Finanzhilfen direkt oder indirekt auf Kosten der Gewinne, die insbesondere den Pionierunternehmen nach Abzug der Steuern. verbleiben. Insofern können Innovations-Subventionen $a b$ einem gewissen Ausmasse wirtschaftliches Wachstum sogar bremsen. Auf diese Umverteilungs-Problematik kann in diesem Artikel nicht näher eingegangen werden. $V g l$. dazu V.Kind, Forschung und Wettbewerbsfähigkeit, in: Die Volkswirtschaft 2/1977

29) Dazu: Philippe Aydalot, Dynamique spatiale et développement inégal, Paris 1976, v.a. Seite $137 \mathrm{ff}$. und David w. Heal, Ownership, Control and Location Revisions, the case of the British Steel Industry since 1945, in: F.E. Jan Hamilton (Ed.), Spatial Perspectives 42) on Industrial Organization and Decisionmaking, London-New York-Sydney-Toronto 1974

30) Zitat aus: Wilhelm Hill, a.a.O. Seite 19 Teilweise Ausnahmen von dieser Regel sind die vier unternehmerischen Bemühungen um vertikale Strukturen: ASUAG, GWC, SSIH und SGT. Diese Firmen besitzen ein erhebliches Potential, vereinigen sie doch $20 \%$ der mengenund annähernd $40 \%$ der wertmässigen Exporte auf sich.

31) Deutlich wird dies in der brancheninternen Studie der Fédération horlogère: "L'horlogerie suisse dans la vision des 10 ans à venir", a.a.O. Seite 41 ff.

32) So der Titel einer Artikelserie über Innovation in der Frankfurter Allgemeinen 1977

33) Vgl. die Studie der Fédération horlogère, a.a.O. Seite 51 ff.

34) Eine entsprechende Studie mit gesamtschweizerischer Betrachtungsweise ist im Nationalen Forschungsprogramm REGIONALPROBLEME IN DER SCHWEIZ vorgesehen.

35) Dazu der Artikel: "SSIH: Siber Hegner als neuer Partner" in Finanz und wirtschaft Nr. 43 vom 4.6. 1977 Seite 21

36) Beispielsweise die Konzentration der headquarter-Funktionen der Mondaine watch Co. in Zürich
37) Eine Tendenz in dieser Richtung ist alich in der Schweiz spürbar; in der BRD zeigen entsprechende Analysen ein Schwergewicht strukturerhaltender Massnahmen trotz deutlicher Ausrichtung der gesetzlichen Grundlagen auf Ziele der Strukturanpassung und -gestaltung. Vgl. dazu Gerhard Voss, Sektorale Strukturpolitik, Anspruch und Praxis, Köln 1977

38) Vgl. dazu: Hugo Allemann, Möglichkeiten und Ziele kantonaler Wirtschaftspolitik, in: Festgabe Franz Josef Jäger, Solothurn 1973; Hans Elsasser, Massnahmen zur Förderung der Industrieansiedlung in den Kantonen, in: DISP Nr. 28, Zürich April 1973; sowie Anmerkung 17

39) Aehnlicher Meïnung ist Georges Fischer, Regionale Anliegen an ein Strukturleitbild Schweiz, in: Wirtschaftspolitische Mitteilungen $8 / 1976$

40) Dies ist auch die Zielsetzung eines gegenwärtig in parlamentarischer Beratung stehenden Bundesbeschlusses für Finanzierungsbeihilfen an wirtschaftlich gefährdete Regionen. Er tritt voraussichtlich Anfang 1979 in Kraft.

41) Zum Zusammenhang von Forschung und Entwicklung mit Innovationsfähigkeit vgl. den Artikel von Ernst-Jürgen Horn, a.a.O. Eine entsprechende Aktivität des Bundes besteht seit einige Jahren im Rahmen der Kommission zur Förderung der angewandten Forschung. Die verfügbaren Finanzmittel sind jedoch eng beschränkt. Vgl. auch Ronge V., Forschungspolitik als Strukturpolitik, München 1977

2) "Les moyens doivent répondre aux deux critères essentiels de la recherche: la continuité et la masse". Zitat aus Maillat/Juvet/Kunz a.a.o. I Seite 25. Die Bedeutung dieser innovationsfördernden Atmosphäre kann in einem weiteren Blickfeld der Bedeutung des Grades an sozialer Integration gleichgesetzt werden. Dieser scheint für Phasen des wirtschaftlichen Aufund Abschwungs bereits im 19. Jahrhundert entscheidend gewesen zu sein; vgl. H. Siegenthaler, Kapitalbildung und sozialer wandel in der Schweiz 1850 - 1914; in Jahrbücher für Nationalökonomie und Statistik, Band 193, Heft 1 (1978)

43) Fédération horlogère suișse, a.a.O. Seite $54 \mathrm{ff}$.

44) Beispielsweise in Studienkommission für Preis-, Kosten- und Strukturfragen, a.a.O. und westfälische Wilhelms-Universität (Sonderforschungsbereich Raumordnung und Raumwirtschaft, Münster), Implikationen des Strukturwandels der Wirtschaft für die Steuerung der räumlichen Entwicklung, Münster 1977

45) Auf strukturpolitische Grenzen macht aufmerksam F. Mühlemann, Möglichkeiten und Grenzen strukturpolitischer Eingriffe des Bundes, in: Schweiz. Zeitschrift für Volkswirtschaft und Statistik $1 / 1974$ 